\title{
Assured and Coherent Sharing of Healthcare Data in Cloud using Cryptography
}

\author{
S. Vidhya, V. Kalaivani
}

\begin{abstract}
The Healthcare information system (HIS) is a compendious system which is developed with a help of various healthcare employees. This information system helps to manage the entire working system of a hospital in paperless environment. Here all the data's related to a patient's are stored in a single database. It offers various benefits to a hospital, but fails to provide the better privateness and security to the data's stored in healthcare database. Various cryptography algorithms are used by the users to share the health information in cloud, which provides secured data sharing. But still, data breeches is the main problem in cloud. This paper proposes a framework for secure sharing of healthcare data in cloud.
\end{abstract}

Index Terms: Database, Cloud, Healthcare information system, Paperless environment, Security.

\section{INTRODUCTION}

The Healthcare organization having several problems in storing and sharing the health data [1]. When data quickly and also it requires more time and storage space to get and stock the data [2].The main concern is to protect the privateness and secrecy of patient's information [3]. To overcome these problems, several techniques are evolving to manage the healthcare database [4]. When the data's are stored in HIS, it is easy to retrieve the data's easily when compared to the traditional method.

The Healthcare Information system plays a vital role in controlling the healthcare related Information [6]. A Healthcare data mainly focus on the administrative demand of the healthcare center. The first HIS was introduced in the year 1960 and now it the alteration of paperless environment. The HIS helps to understand the significance of different departments present in the healthcare center, [7], [8]. By a simple click of mouse the professional can receive any information about the patient. The HIS is a best software solution developed with the partnership of various clinical officers to improve the patient data privacy[9].If it is not flexible then the changes in technology will affect the growth of hospital. Modern hospital information system typically uses fast computers which are connected with optimized network.

The healthcare information system holds the entire health history of a patient. This information should be stored in a well secured place and it must allow only the authenticated person to access the health record [10]. The healthcare providers can communicate in internal and external fashion. With the help of HIS the healthcare providers can access the

Revised Manuscript Received on July 22, 2019.

Mrs Vidhya S, Assistant Professor ,Computer Science \& Engineering, Ponjesly College of Engineering, Nagercoil, India.

Dr V Kalaivani, Professor, National Engineering College, India. record in faster and better way [11]. It also helps the patients to know about their surgical methods by the help of animation and pictorial representation. If a hospital want to shift a patient from their hospital to another then the medical history of that patient is moved from their HIS to other HIS for better performance. The medical information stored by these facilities can always be easily retrieved.Within the organization, a HIS can refer some other system, which helps store and transfer the patient's health data [12]. The HIS manage all the information effectively, and those information is used by the professionals to do their work in systematic manner. The HIS required various requirements like factual, planning (decision about hospital information) and documentation requirements [13].

This study carries out with a importance of healthcare information system and also the various security issues. By this, the researchers can able to know the importance of healthcare system and helps them to do many researches in providing security to HIS. It also helps to implement the various technologies in healthcare organization which provides the patient's data in standard format and also helps the software developer to know the importance of HIS and various security issues[14].

A structure of healthcare information system [15] is shown in the below figure

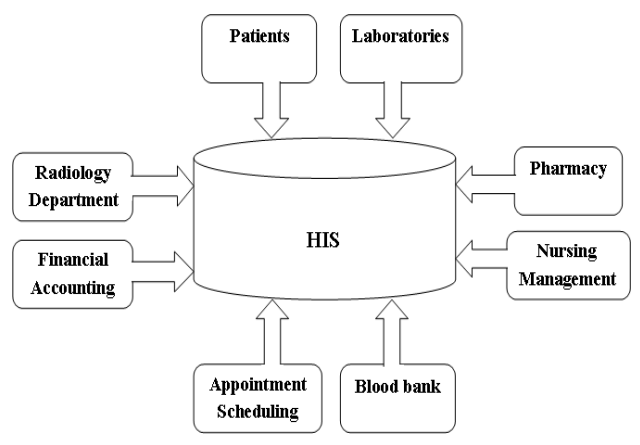

Figure 1: HIS Model

The various components present in figure 1 is described below

Patients: Patient is used to gather, store and handle the information about patients.

Laboratories: HIS manages the various lab related information like patient's medical testing including hematology, chemistry, immunology and microbiology.

Pharmacy: It stores all the information's related to pharmacy. Activities involved in pharmacy IS are prescription management, inventory management, patient's drug profile, clinical screening etc [16].

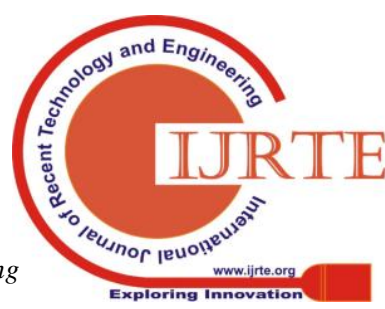


Financial and Accounting: The aim of the hospital is to provide better treatment to patients and not for financial gain, they do obtain the cost from all the activities. It manages all the business aspects of the hospital, and ease the difficult task that faces hospitals.

Radiology: Radiology information system is a system, which helps to manage the visual representation of the interior body an associated data. Within the radiology department, RIS monitor a patient's complete workflow and inform the report to hospital.

Appointment Scheduling: It captures the entire information of patients and helps to schedule the appointments. In patient registration module scheduling, rescheduling and manipulation can done easily.

Blood bank: Computer system designed to store the information related to blood donation in accurate manner. It maintains the details about the donor and the recipients. This information system records the important information such as availability of blood, matching between donor's and recipient's blood groups and the transfusion reactions of blood. Also it records the interactions with other blood banks. Nursing IS: Nursing information system are computer system used to maintain the nursing record, which helps the nurses to improve the patient care [17].

Administration: The hospital administration system covers patients registration, doctor scheduling, consultation, cash and transaction management, pharmacy catalogue, diagnosis result etc..

The quality of hospitals can be improved with the help of this HIS. Different technologies are used by various countries to improve to the healthcare services. But still these countries facing different problem to implement these technologies [18]. Due to high startup cost, country like japan failed to implement this EHR. Sometimes it is difficult to take decision due to technical and behavioral barriers.

The major issue in HIS is data store and data transmission [16]. If the number of people in HIS increases, the storage capacity of HIS should also increase, which also leads to increase the hospital cost, says that different types of software and hardware are integrated to implement HIS is very expensive.

The uses of HIS also have several benefits that include more accurate, reduce time and storage space, reduce human error, easy to access and also it improves the health services. According to, HIS security is the most main issue, because the data stored in HIS are related to patient which are very sensitive one.

\section{A. HIPAA}

In 1996, the HIPAA, sanction a law in to design the software to protect the hospital data security and confidentiality [18]. The law is divided in to two which focuses on portability and administrative simplification. The portability ensure that the individual patients can carry their insurance copy from one place to another administrative simplification focuses on the data transmission and providing security and data privateness.

HIPAA regulations can apply to all healthcare providers to guard the record which contains the complete information about the patient. According to Ponemon institute report, now the rate of data violation is $\$ 3.8$ million from the year 2013 .

According to the review, web-borne mal-ware attack is the main reason to most of the data breaches in hospital, where the malicious hackers append the malicious code to the internet web page using java script. When the users visit that infected webpage, the malware affects the user's PC.

\section{NEED OF SECURITY IN HIS}

In HIS, the data leakage is the main problem. The hackers have complete power to hack the sensitive data. In many cases the hospital data's has been accessed by some unauthorized person, which creates some unbelief in the minds of physicians and patients [19]. If one unauthorized person get that hospital information, then the result will be enormous. The breach of hospital information can destroy the patient's finance, credit and reputation. Because of this reason only, now various companies have worked hard to implement security in health database. The State of Health Privacy project describes certain rules to handle the healthcare data's.

Nowadays, Hospitals facing a set of challenges to protect their information. These digitized health records are extremely valuable to offender, while hospitals and other organizations are still learning how to protect them. The people from hospitals; research center, insurance companies and other related organizations need access to patient health record to do their jobs. The sensitive data present in hospitals spread across number of devices like desktops, laptops, mobile devices and specialized devices for inputting health record. According to most recent healthcare report 68 percent of hospital data breaches were due to device theft or loss. Security in HIS ensures the patients that their information remains confidential and only the authorized person can access their information.

\section{DATA SECURITY ISSUES}

Data security helps to protect the data from unknown users. Security is very important for hospital records, so the medical practitioners from various countries working toward implementing electronic health record privacy. The threats can be arise from both interior and exterior surroundings of the Hospital

Interior Environment: The hospital's internal environment refers to the people working inside the hospital and conditions inside the hospital that are generally under the control of the hospital.

Exterior Environment: The exterior environment of a hospital refers the people outside the hospital who can cause change inside the hospital for the most part, beyond the control of hospital. Even though the problem occurs in the external environment of the hospital, it can have a significant influence on the growth of the hospital. It is necessary for the health professionals to have a monitoring over the external factors and take necessary actions against it.

The data security helps to provide complete protection to patient's privacy and secrecy. Patient secrecy refers to the protection of patient data from errors and the privacy of the patient refers the protection of health records from unauthorized users. The security system must ensure that the information present in the system can be accessed only by the person who having the complete authorization over the records. Security in HIS is very important because it provides confidentiality and privacy to patients. Although HIS security is very important, the research literature lacks to identify the various threats affecting the patient privacy and safety. 
Many professionals carry out the study in information security and found the various issues related to healthcare information system [20]. Cloud computing technology is used to stock the healthcare information, where we can get back the information at very fast rate. Even though it is easy to get back the information at very fast rate, the security is the main barrier in cloud computing [21].

\section{SECURITY THREATS IN HIS}

The main 5 security threats for hospital organizations are given below

\section{A. Mobile Devices:}

Nowadays, various types of mobile devices are used by healthcare professionals, patients and other people. The proper communication is done through network, which helps to ensure the quality of patient care. The different version of Operating system may affect these devices

\section{B. Embedded Devices:}

The mobile phones are used with the help of Wi-Fi connectivity to observe and control the productivity in easier manner.

\section{Virtualization:}

In virtualization, many applications can run on a single server. Virtualization technique is used in healthcare organization to reduce the management cost and to make use of all the flexibility offered by the virtualized desktops. But unfortunately, it leads to many threats, due to increase in number of customer.

\section{Virus Escalation:}

The viruses can spread easily through social media sites, where the healthcare users may have their own secure data.

\section{E. IT Becomes Consumer Friendly:}

Healthcare organizations facing more problem, due to the adoption of own device.[30].

\section{SECURITY CHALLENGES IN HIS}

In hospital, now the individuals can access their information via online portals itself. The evolvement of new technology and legislation also creates the various changes in security threats. The various obstacles in healthcare information system are lack of return on investment, cost, issue in usage, security and privacy [23].

The success/failure rate of healthcare information system is decided with the help of the gap present in between design and real attainment. According to, the exchange of health data should produce a value of dollar 77.8 billion in a year. In healthcare organization the cost of HIS remains as a major concern, this is due to high Capital investment and low return on investment. The five biggest data security challenges [24], [25] are

\section{A. Exchange of Medical record}

Health Information Exchange (HIEs) is used to help doctors to share the health information. By using network, large amount of information can be transformed from one place to another. The sharing of information between multiple providers creates a opportunity to hackers to hack the data. HITECH provides incentives for health record adoption, in which the providers are responsible to notify the patients about the breach of medical record.

\section{B. Technology error created by user}

The error created by the patient is also one of the data hazard. When the patient gets the data from the health professionals, and stores that data in unencrypted folders or sends the information to others through email may help the hackers to get the information

\section{Rise in Hacktivism}

The HIS stores the patients sensitive data including the social security number. Hackers can launch the DDoS attack on the hospital website as an act of "hacktivism". Hacktivism is the act of breaking in to the computer system for some purposes.

\section{Evolvement of cloud and mobile technology in HIS}

Now cloud is used to stock the healthcare information. HITECH mandates the encryption of health record, which is the serious problem in cloud. The usage of mobile technology also leads to the serious threats. The hospitals must keeping careful watch for possible changes and difficulties to ensure that use of mobile app and cloud isn't violating the act of HIPAA.

\section{E. Virtualization}

Virtualization allows the servers to run multiple applications which reduce the cost associated with the energy. Hospitals use this virtualization software to perform their own operations. Since large number of users move to this virtualization environment there is a possibility to threats

\section{SOLUTION SCHEME}

The healthcare data contains two different types of data. One is restricted data, which can be accessed by only authorized people and another one is general data. The third party server must provide more security to this general data. In this scheme, the owner of the healthcare data have to encrypt the data using some cryptographic algorithm. When the encrypted data moves inside proxy server, again it will be re-encrypted using asymmetric encryption algorithm. If a user wants to access any data from cloud, then ACL of the user will be checked. If the key and access permission get granted, then he/she can decrypt the data.

\section{CONCLUSION}

It is very important for cloud server to develop comprehensive software to protect the patient health records from the security violations. The healthcare information system collects tremendous information about the hospital and it is very important that the patient should feel secure to provide their information. Assured and coherent data sharing can be achieved using this solution framework. This paper covers the importance of healthcare database system and the technical issues present in the HIS. In future, it is very important to provide a strong security to hospital information system due to the evolution of new technologies in medical field.

\section{REFERENCES}

[1] Balaraman, P., and Kosalram, K, Ehospital Management \& Hospital Information Systems Changing Trends, International Journal Information Engineering and Electronic Business.

[2] Xiaokui Shu, Danfeng Yao, Member, IEEE, and Elisa Bertino, Fellow, IEEE, Privacy-Preserving Detection of Sensitive Data Exposure, IEEE Transactions on Information Forensics and Security. 
[3] Computer Science and Telecommunications Board, Networking Health: Prescriptions for the Internet, 2000.

[4] M. Cashen, P. Dykes and B. Gerber. 2004, EHealth Technology and Internet Resources:Barriers for Vulnerable Populations, Journal of Cardiovascular NursingVol. 19, No. 3, pp 209- 214 () 2004 Lippincott Williams \& Wilkins, Inc.

[5] Wei, X. (2011). Hospital Information System Management and Security Maintenance, Computing and Intelligent Systems Communications in Computer and Information Science.

[6] Ajit Appari and M. Eric Johnson, Information security and privacy in healthcare: current state of research, Int. J. Internet and Enterprise Management.

[7] Biomedical informatics Ltd, Hospital Information Systems, Retrieved from http://www.biohealthmatics.com/techn ologies/intsys.aspx on July 14, 2011.

[8] Health Privacy Project at www.healthprivacy.org/info-urlnocat2304info- url nocat.html.

[9] D. Masys, D. Baker, A. Butros, K.E. Cowles, Giving patients access to their medical records via the internet: the PCASSO experience, Journal of American Medical Informatics Association.

[10] T. Greenhalgh, S. Hinder, K. Stramer, T. B ratan, J. RussellAdoption, non-adoption, and abandonment of a personal electronic health record: case study of HealthSpace BMJ.

[11] www.sciencedirect.com/topics/medicineand-dentistry/protected-health-information

[12] Prajakta Pawar, Sushopti Gawade, Heuristic Walkthrough Usability Evaluation of Electronic Health Record with a Proposed Security Architecture International Journal of Innovative Research in Computer Science \& Technology.

[13] www.groups.csail.mit.edu/medg/courses/6 872/96/notes/sheldon.html

[14] O. Osunmuyiwa and A. H. Ulusoy, Wireless security in mobile health, Telemedicine and e-health.

[15] Maslin Masrom, Ailar Rahimly, Overview of Data Security Issues In Hospital Information System, Pacific Asia Journal of the Association for Information Systems.

[16] S. Kahn and V. Sheshadri, Medical Record Privacy and Security in a Digital Environment, IT Professional.

[17] Y.A. AL-nassar, A.M. Mohd and O. S. Wan. 2011. Overcoming challenges to use Electronic Medical Records System (EMRs) in Jordan Hospitals, IJCSNS International Journal of Computer Science and Network Security.

[18] "HealthInsurance Reform:Security Standards,"HHS, Washington, 2003 http://www.cms.hhs.gov/SecurityStandard/Do wnloads/securityfinalrule.pdf,.

[19] Bundesamt für Sicherheit in der Informationstechnik, "IT-Grundschutz- Kataloge," BSI, Köln, 2005.

[20] David Houlding, MSc, CISSP: Health Information at Risk: Successful Strategies for Healthcare Security and Privacy : Healthcare IT Program Of ce Intel Corporation, white paper 2011.

[21] Hajrahimi, N., Dehaghani, S. M. H., and Sheikhtaheri, A, Health Information security: A Case Study of Tree Selected Medical Centers, Acta Informatica Medica.

[2] Anderson, J. G. Clearing the way for physicians' use of clinical information systems. Communications of the ACM.

23] G. Arash and Z. Shukur, Security Challenges and Success Factors of Electronic Healthcare System, University Kebangsaan Malaysia.

24] Eystein Mathisen, Security Challenges and Solutions in Cloud Computing, in: International Conference on Digital Ecosystems and Technologies.

[25] T. Greenhalgh, S. Hinder, K. Stramer, T. B ratan, J. RussellAdoption, non-adoption, and abandonment of a personal electronic health record: case study of HealthSpace BMJ.
AUTHORS PROFILE

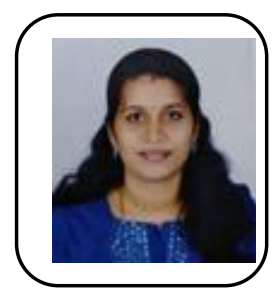

Vidhya $\mathbf{S}$ received her Master of Engineering from Anna University, Chennai in 2012, her Bachelor of Engineering from Anna University, Chennai in 2010. She is currently a Assistant Professor at Ponjesly College of Engineering, Nagercoil. Her current research interests include, cryptography, cloud security and computer security.

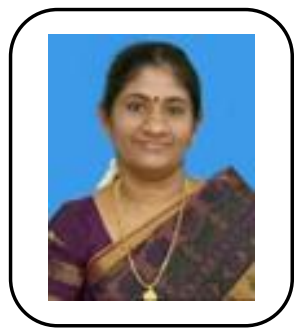

Dr.V.Kalaivani (Velayutharaman Kalaivani) is working as Professor in Department of Computer Science and Engineering, National Engineering College, Kovilpatti,Tamilnadu,India. She received her $\mathrm{Ph} . D$. degree from Anna University, Chennai in 2011. Then she obtained her Master's degree in Computer science and Engineering from Anna University in 2002, Chennai and Bachelor's degree from the Madurai Kamaraj University in 1996. She has 20 years of teaching experience and 15 years of research experience. She has published 22 international journals and has presented her research work in 23 International conferences and 20 National conferences. Her research interests are in Web Mining, Medical Image Analysis and Data Mining. She is a Life Member in ISTE and MIE in Institution of Engineers (India) 